\title{
X-ray screening identifies active site and allosteric inhibitors of SARS-CoV-2 main protease
}

\begin{abstract}
Sebastian Günther ${ }^{1 *}$, Patrick Y. A. Reinke ${ }^{1}+$, Yaiza Fernández-García ${ }^{2}$, Julia Lieske ${ }^{1}$, Thomas J. Lane' ${ }^{1}$ Helen M. Ginn ${ }^{3}$, Faisal H. M. Koua ${ }^{1}$, Christiane Ehrt ${ }^{4}$, Wiebke Ewert ${ }^{1}$, Dominik Oberthuer', Oleksandr Yefanov', Susanne Meier ${ }^{5,6}$, Kristina Lorenzen ${ }^{7}$, Boris Krichel ${ }^{8}$, Janine- $^{1}$ Denise Kopicki' ${ }^{8}$, Luca Gelisio' ${ }^{1}$, Wolfgang Brehm ${ }^{1}$, Ilona Dunkel ${ }^{9}$, Brandon Seychel10 ${ }^{10}$, Henry Gieseler ${ }^{5,6}$, Brenna Norton-Baker ${ }^{11,12}$, Beatriz Escudero-Pérez ${ }^{2}$, Martin Domaracky ${ }^{1}$, Sofiane Saouane ${ }^{13}$, Alexandra Tolstikova ${ }^{1}$, Thomas A. White ${ }^{1}$, Anna Hänle ${ }^{1}$, Michael Groessler', Holger Fleckenstein ${ }^{1}$, Fabian Trost' ${ }^{1}$, Marina Galchenkova ${ }^{1}$, Yaroslav Gevorkov ${ }^{1,14}$, Chufeng Li ${ }^{1}$, Salah Awel ${ }^{1}$, Ariana Peck ${ }^{15}$, Miriam Barthelmess $^{1}$, Frank Schlünzen ${ }^{1}$, P. Lourdu Xavier ${ }^{1,11}$, Nadine Werner ${ }^{16}$, Hina Andaleeb ${ }^{16}$, Najeeb Ullah ${ }^{16}$, Sven Falke $^{16}$, Vasundara Srinivasan ${ }^{16}$, Bruno Alves França ${ }^{16}$, Martin Schwinzer ${ }^{16}$, Hévila Brognaro ${ }^{16}$, Cromarte Rogers ${ }^{5,6}$, Diogo Melo ${ }^{5,6}$, Joanna J. Zaitseva-Doyle ${ }^{5,6}$, Juraj Knoska', Gisel E. Peña-Murillo ${ }^{1}$, Aida Rahmani Mashhour ${ }^{1}$, Vincent Hennicke ${ }^{1}$, Pontus Fischer ${ }^{1}$, Johanna Hakanpää̈ ${ }^{3}$ Jan Meyer $^{13}$, Philip Gribbon $^{17}$, Bernhard Ellinger ${ }^{17}$, Maria Kuzikov ${ }^{17}$, Markus Wolf ${ }^{17}$, Andrea R. Beccari' ${ }^{18}$, Gleb Bourenkov ${ }^{19}$, David von Stetten ${ }^{19}$, Guillaume Pompidor $^{19}$, Isabel Bento ${ }^{19}$, Saravanan Panneerselvam ${ }^{19}$, Ivars Karpics ${ }^{19}$, Thomas R. Schneider ${ }^{19}$, Maria Marta Garcia-Alai $^{19}$, Stephan Niebling ${ }^{19}$, Christian Günther ${ }^{19}$, Christina Schmidt ${ }^{7}$, Robin Schubert ${ }^{7}$, Huijong Han ${ }^{7}$, Juliane Boger ${ }^{20}$, Diana C. F. Monteiro ${ }^{21}$, Linlin Zhang ${ }^{20,22}$, Xinyuanyuan Sun ${ }^{20,22}$, Jonathan Pletzer-Zelgert ${ }^{4}$, Jan Wollenhaupt ${ }^{23}$, Christian G. Feiler ${ }^{23}$, Manfred S. Weiss ${ }^{23}$, EikeChristian Schulz ${ }^{11}$, Pedram Mehrabi ${ }^{11}$, Katarina Karničar ${ }^{24,25}$, Aleksandra Usenik ${ }^{24,25}$, Jure Loboda $^{24}$, Henning Tidow $^{5,26}$ Ashwin Chari $^{27}$, Rolf Hilgenfeld ${ }^{20,22}$, Charlotte Uetrecht ${ }^{8}$, Russell Cox $^{28}$, Andrea Zaliani ${ }^{17}$, Tobias Beck ${ }^{5,10}$, Matthias Rarey ${ }^{4}$, Stephan Günther ${ }^{2}$, Dusan Turk $^{24,25}$, Winfried Hinrichs ${ }^{16,29}$, Henry N. Chapman ${ }^{1,5,30}$, Arwen R. Pearson ${ }^{5,6}$, Christian Betzel ${ }^{5,16}$, Alke Meents ${ }^{1 *}$

${ }^{1}$ Center for Free-Electron Laser Science, DESY, Notkestr. 85, 22607 Hamburg, Germany. ${ }^{2}$ Bernhard Nocht Institute for Tropical Medicine, Bernhard-Nocht-Str. 74,20359 Hamburg, Germany. ${ }^{3}$ Diamond Light Source Ltd. Diamond House, Harwell Science and Innovation Campus, Didcot, OX11 ODE, UK. ${ }^{4}$ Universität Hamburg, Center for Bioinformatics, Bundesstr. 43, 20146 Hamburg, Germany. ${ }^{5}$ Hamburg Centre for Ultrafast Imaging (CUI), Universität Hamburg, Luruper Chaussee 149, 22761 Hamburg, Germany. ${ }^{6}$ Universität Hamburg, Institut für Nanostruktur- und Festkörperphysik, Luruper Chaussee 149, 22761 Hamburg, Germany. ${ }^{7}$ European XFEL GmbH. Holzkoppel 4, 22869 Schenefeld, Germany. ${ }^{8}$ Heinrich Pette Institute, Leibniz Institute for Experimental Virology, Martinistr. 52, 20251 Hamburg, Germany. ${ }^{9}$ Max Planck Institute for Molecular Genetics, Ihnestr. 63-73, 14195 Berlin, Germany. ${ }^{10}$ Universität Hamburg, Department of Chemistry, Institute of Physical Chemistry, Grindelallee 117,20146 Hamburg, Germany. ${ }^{11}$ Max Planck Institute for the Structure and Dynamics of Matter, Luruper Chaussee 149, 22761 Hamburg, Germany. ${ }^{12}$ Department of Chemistry, UC Irvine, Irvine, CA 92697-2025, USA. ${ }^{13}$ Deutsches Elektronen-Synchrotron (DESY), Photon Science, Notkestr. 85, 22607, Hamburg, Germany. ${ }^{14}$ Vision Systems, Hamburg University of Technology, 21071 Hamburg, Germany. ${ }^{15}$ Division of Biology and Biological Engineering, California Institute of Technology, Pasadena, CA 91125, USA.

${ }^{16}$ Universität Hamburg, Department of Chemistry, Institute of Biochemistry and Molecular Biology and Laboratory for Structural Biology of Infection and Inflammation, c/o DESY, 22607 Hamburg, Germany. ${ }^{17}$ Fraunhofer Institute for Translational Medicine and Pharmacology (ITMP) and Fraunhofer Cluster of Excellence for Immune Mediated Diseases (CIMD), Schnackenburgallee 114, 22525 Hamburg, Germany. ${ }^{18}$ Dompé Farmaceutici SpA, 67100 L'Aquila, Italy. ${ }^{19}$ EMBL Outstation Hamburg, c/o DESY, Notkestr. 85, 22607 Hamburg, Germany. ${ }^{20}$ Institute of Molecular Medicine, University of Lübeck, 23562 Lübeck, Germany. ${ }^{21}$ Hauptmann Woodward Medical Research Institute, 700 Ellicott Street, Buffalo, NY, 14203, USA. ${ }^{22}$ German Center for Infection Research (DZIF), Hamburg-Lübeck-Borstel-Riems Site, University of Lübeck, 23562 Lübeck, Germany. ${ }^{23} \mathrm{Helmholtz}$ Zentrum Berlin, Macromolecular Crystallography, Albert-Einstein-Str. 15, 12489 Berlin, Germany. ${ }^{24}$ Department of Biochemistry and Molecular and Structural Biology, Jozef Stefan Institute, Jamova 39, 1000 Ljubljana, Slovenia. ${ }^{25}$ Centre of excellence for Integrated Approaches in Chemistry and Biology of Proteins (CIPKEBIP), Jamova 39, 1000 Ljubljana, Slovenia. ${ }^{26}$ Universität Hamburg, Department of Chemistry, Institute of Biochemistry and Molecular Biology, Martin-Luther-KingPlatz 6, 20146 Hamburg, Germany. ${ }^{27}$ Research Group for Structural Biochemistry and Mechanisms, Department of Structural Dynamics, Max Planck Institute for Biophysical Chemistry, Am Fassberg 11, 37077 Göttingen, Germany. ${ }^{28}$ Institute for Organic Chemistry and BMWZ, Leibniz University of Hannover, Schneiderberg 38 , 30167 Hannover, Germany. ${ }^{29}$ Universität Greifswald, Institute of Biochemistry, Felix-Hausdorff-Str. 4, 17489 Greifswald, Germany. ${ }^{30}$ Universität Hamburg, Department of Physics, Luruper Chaussee 149, 22761 Hamburg, Germany.
\end{abstract}

*Corresponding author. Email: sebastian.guenther@desy.de (S.G.); alke.meents@desy.de (A.M.)

†These authors contributed equally to this work.

The coronavirus disease (COVID-19) caused by SARS-CoV-2 is creating tremendous human suffering. To date, no effective drug is available to directly treat the disease. In a search for a drug against COVID-19, we have performed a high-throughput X-ray crystallographic screen of two repurposing drug libraries against the SARS-CoV-2 main protease $\left(\mathrm{M}^{\text {pro }}\right)$, which is essential for viral replication. In contrast to commonly applied X-ray fragment screening experiments with molecules of low complexity, our screen tested already approved drugs and drugs in clinical trials. From the three-dimensional protein structures, we identified 37 compounds that bind to $\mathrm{M}^{\text {pro. }}$. In subsequent cell-based viral reduction assays, one peptidomimetic and six non-peptidic compounds showed antiviral activity at non-toxic concentrations. We identified two allosteric binding sites representing attractive targets for drug development against SARS-CoV-2. 
Infection of host cells by SARS-CoV-2 is governed by the complex interplay of molecular factors from both the host and the virus $(1,2)$. Coronaviruses are RNA-viruses with a genome of approximately 30,000 nucleotides. The viral open-reading frames are expressed as two overlapping large polyproteins, which must be separated into functional subunits for replication and transcription activity (1). This proteolytic cleavage is primarily accomplished by the main protease $\left(\mathrm{M}^{\mathrm{pro}}\right)$, also known as $3 \mathrm{C}$-like protease $3 \mathrm{CL}^{\text {pro }}$ or nsp5. $\mathrm{M}^{\text {pro }}$ cleaves the viral polyprotein pplab at eleven distinct sites. The core cleavage motif is Leu-Gln $\downarrow$ (Ser/Ala/Gly) (1). $\mathrm{M}^{\text {pro }}$ possesses a chymotrypsin-like fold appended with a C-terminal helical domain, and harbors a catalytic dyad comprised of Cys145 and His41 in its active site, which is formed by four major pockets that are labeled according to their position relative to the scissile bond of the substrate (Fig. 1) (I). The active site is located in a cleft between the two N-terminal domains of the three-domain structure of the monomer, while the C-terminal helical domain is involved in regulation and dimerization of the enzyme (Fig. 1A). Due to its central involvement in virus replication, $\mathrm{M}^{\text {pro }}$ is recognized as a prime target for antiviral drug discovery and compound screening activities aiming to identify and optimize drugs which can tackle coronavirus infections (3). Indeed, a number of recent publications confirm the potential of targeting $\mathrm{M}^{\text {pro }}$ for inhibition of virus replication $(1,2,4)$.

In order to find drug candidates against SARS-CoV-2, we performed a large-scale X-ray crystallographic screen of $\mathrm{M}^{\text {pro }}$ against two repurposing libraries containing 5953 unique compounds from the "Fraunhofer IME Repurposing Collection" and the "Safe-in-man" library from Dompé Farmaceutici S.p.A. (5).

In contrast to crystallographic fragment-screening experiments, repurposing libraries are chemically more complex (fig. S1A) $(6,7)$. Thus they likely bind more specifically and with higher affinity (8). Due to the higher molecular weights, we performed co-crystallization experiments at a physiological $\mathrm{pH}$-value of 7.5 instead of compound soaking into native crystals (9).

From the 5953 unique compounds in our screen, we obtained X-ray diffraction datasets from 2381 unique compounds, which were subjected to automated structure refinement followed by cluster analysis (10) and pan dataset density analysis (PanDDA) (11) (table S1). We observed additional electron-density, indicating binding to $\mathrm{M}^{\text {pro }}$, for 43 compounds, which were classified as hits, representing 37 unique compounds (tables S1, S2, and S3). From these, the binding mode could be unambiguously determined for 29 molecules (Fig. 1A and table S4). The majority of hits were found in the active site of the enzyme. Of the 16 active-site binders, six covalently bind as thioethers to Cys145, one compound binds covalently as a thiohemiacetal to Cys145, one is zinc-coordinated and eight bind non-covalently. The remaining 13 compounds bind outside the active site at various locations (Fig. 1A).

Of the 43 hits from our X-ray screen, 37 compounds were available in quantities required for testing their antiviral activity against SARS-CoV-2 in cell assays (table S2). Nine compounds, that reduced viral RNA (vRNA) replication by at least two orders of magnitude in Vero E6 cells (fig. S2), were further evaluated to determine the effective concentrations that reduced not only vRNA but also SARS-CoV-2 infectious particles by $50 \%\left(\mathrm{EC}_{50}\right)$ (Fig. 2). Additionally, AT7519 and ifenprodil, which showed slightly lower vRNA-level reduction, were included due to their distinct binding sites outside of the active site. From these eleven, seven compounds (AT7519, calpeptin, ifenprodil, MUT056399, pelitinib, tolperisone, triglycidyl isocyanurate) exhibited at least one hundredfold reduction in infectious particles in combination with either selectivity indices $\left(\mathrm{SI}=\mathrm{CC}_{50} / \mathrm{EC}_{50}\right.$ ) greater than five or no cytotoxicity in the tested concentration range and are considered antivirally active (table S5).

In the following we focus on a more detailed description of the eleven compounds analyzed in the secondary screen, which are grouped according to their different binding sites. The remaining hits are described in the supplementary text and figs. S3 to S5.

Tolperisone, HEAT and isofloxythepin bind covalently to the active site. Tolperisone is antivirally active $\left(\mathrm{EC}_{50}=19.17\right.$ $\mu \mathrm{M})$ and shows no cytotoxicity $\left(\mathrm{CC}_{50}>100 \mu \mathrm{M}\right)$ (Fig. 2), whereas HEAT $\left(\mathrm{EC}_{50}=24.05 \mu \mathrm{M}, \mathrm{CC}_{50}=55.42 \mu \mathrm{M}\right)$ and isofloxythepin $\left(\mathrm{EC}_{50}=4.8 \mu \mathrm{M}, \mathrm{CC}_{50}=17 \mu \mathrm{M}\right)$ show unfavorable cytotoxicity. For all three compounds, only breakdown products are observed in the active site. Tolperisone and HEAT are $\beta$-aminoketones, but we only observe the part of the drug containing the ketone (2,4'-dimethylpropiophenone and 2-methyl-1-tetralone), while the remaining part with the amine group is missing. The breakdown product binds as a Michael acceptor to the thiol of Cys145, independently confirmed for HEAT by mass spectrometry (fig. S6 and table S6). The decomposition of tolperisone and HEAT was detected in both the crystallization and cell culture conditions (fig. S7) and is reported to be pH-dependent (12). The parent compounds can be regarded as pro-drugs $(13,14)$. In the X-ray structures the aromatic ring systems of tolperisone (Fig. 3A) and HEAT (Fig. 3B) protrude into the S1 pocket and form van der Waals contacts with the backbone of Phe140 and Leu141 and the side chain of Glu166. In addition, the keto group accepts a hydrogen bond from the imidazole side chain of His163. Tolperisone is used as a skeletal muscle relaxant (15). The X-ray structure suggests that isofloxythepin binds similarly as a fragment to Cys145 (Fig. 3C).

Triglycidyl isocyanurate has antiviral activity $\left(\mathrm{EC}_{50}=\right.$ $30.02 \mu \mathrm{M}, \mathrm{CC}_{50}>100 \mu \mathrm{M}$ ) and adopts a covalent and non- 
covalent binding mode to the active site. In both modes, the compound's central ring sits on top of the catalytic dyad (His41, Cys145) and its three epoxypropyl substituents reach into subsites S1', S1 and S2. The non-covalent binding mode is stabilized by hydrogen bonds to the main chain of Gly143 and Gln166, and to the side chain of His163. In the covalently bound form, one oxirane ring is opened by nucleophilic attack of Cys145 forming a thioether (Fig. 3D). Triglycidyl isocyanurate has been tested as an antitumor agent (16).

Calpeptin shows the highest antiviral activity in the screen $\left(\mathrm{EC}_{50}=72 \mathrm{nM}, \mathrm{CC}_{50}>100 \mu \mathrm{M}\right)$. It binds covalently via its aldehyde group to Cys145, forming a thiohemiacetal. This peptidomimetic inhibitor occupies substrate pockets S1 to S3, similar to the peptidomimetic inhibitors GC-376 $(17,18)$, calpain inhibitors (19), N3 (2), and the $\alpha$-ketoamide 13b (1). The peptidomimetic backbone forms hydrogen bonds to the main chain of His164 and Glu166, whereas the norleucine side chain maintains van der Waals contacts with the backbone of Phe140, Leu141 and Asn142 (Fig. 3E). Calpeptin has known activity against SARS-CoV-2 $\mathrm{M}^{\text {pro }}$ in enzymatic assays (17). The structure is highly similar to the common protease inhibitor leupeptin (fig. S3A), which served as a positive control in our X-ray screen but was not further tested in antiviral assays. In silico docking experiments also suggested calpeptin as a possible $\mathrm{M}^{\text {pro }}$ binding molecule (table $\mathrm{S} 7$ ). Calpeptin also inhibits cathepsin L (20) and dual targeting of cathepsin L and $\mathrm{M}^{\text {pro }}$ is suggested as attractive path for SARS-CoV-2 inhibition (19).

MUT056399 binds non-covalently to the active site $\left(\mathrm{EC}_{50}\right.$ $\left.=38.24 \mu \mathrm{M}, \mathrm{CC}_{50}>100 \mu \mathrm{M}\right)$. The diphenyl ether core of MUT056399 blocks access to the catalytic site consisting of Cys145 and His41. The terminal carboxamide group occupies pocket $\mathrm{S} 1$ and forms hydrogen bonds to the side chain of His163 and the backbone of Phe140 (Fig. 3F). The ethyl-phenyl group of the molecule reaches deep into pocket $\mathrm{S} 2$, which is enlarged by a shift of the side chain of Met49 out of the substrate binding pocket. MUT056399 was developed as an antibacterial agent against multidrug-resistant Staphylococcus aureus strains (21).

Quipazine maleate showed moderate antiviral activity $\left(\mathrm{EC}_{50}=31.64 \mu \mathrm{M}, \mathrm{CC}_{50}>100 \mu \mathrm{M}\right)$. In the X-ray structure, only the maleate counterion is observed covalently bound as a thioether (supplementary text and fig. S3B). Maleate is observed in structures of six other compounds showing no antiviral activity. The observed antiviral activity is thus likely caused by an off-target effect of quipazine.

In general, the enzymatic activity of $\mathrm{M}^{\text {pro }}$ relies on the architecture of the active site, which critically depends on the dimerization of the enzyme and the correct relative orientation of the subdomains. This could allow ligands that bind outside of the active site to affect activity. In fact, we discovered two such allosteric binding sites of $\mathrm{M}^{\text {pro }}$.
Five compounds of our X-ray screen bind in a hydrophobic pocket in the C-terminal dimerization domain (Fig. 4, A and B), located close to the oxyanion hole in pocket S1 of the substrate-binding site. One of these showed strong antiviral activity (Fig. 2). Another compound binds in between the catalytic and dimerization domains of $\mathrm{M}^{\text {pro }}$.

Central to the first allosteric binding site is a hydrophobic pocket formed by Ile213, Leu253, Gln256, Val297 and Cys300 within the C-terminal dimerization domain (Fig. 4A). Pelitinib, ifenprodil, RS-102895, PD-168568 and tofogliflozin all exploit this site by inserting an aromatic moiety into this pocket.

Pelitinib shows the second highest antiviral activity in our screen $\left(\mathrm{EC}_{50}=1.25 \mu \mathrm{M}, \mathrm{CC}_{50}=13.96 \mu \mathrm{M}\right)$. Its halogenated benzene ring binds to the hydrophobic groove in the helical domain which becomes accessible by movement of the Gln256 side chain (Fig. 4A). The central 3-cyanoquinoline moiety interacts with the end of the C-terminal helix (Ser301). The ethyl ether substituent pushes against Tyr118 and Asn142 (from loop 141-144 of the S1 pocket) of the opposing protomer within the native dimer. The integrity of this pocket is crucial for enzyme activity (22). Pelitinib is an amine-catalyzed Michael acceptor (23), developed as an anticancer agent to bind to a cysteine in the active site of the tyrosine kinase epidermal growth factor receptor inhibitor (24). But from its observed binding position it is impossible for it to reach into the active site and no evidence for covalent binding to Cys145 is found in the electron-density maps.

Ifenprodil and RS-102895 bind to the same hydrophobic pocket in the dimerization domain as pelitinib (Fig. 4B; fig. $\mathrm{S} 4, \mathrm{~A}$ and $\mathrm{B}$; and supplementary text). Only ifenprodil ( $\mathrm{EC}_{50}$ $\left.=46.86 \mu \mathrm{M}, \mathrm{CC}_{50}>100 \mu \mathrm{M}\right)$ shows moderate activity. RS$102895\left(\mathrm{EC}_{50}=19.8 \mu \mathrm{M}, \mathrm{CC}_{50}=54.98 \mu \mathrm{M}\right)$ interacts, similar to pelitinib, with the second protomer by forming two hydrogen bonds to the side and main chains of Asn142 while the other compounds exhibit weaker or no interaction with the second protomer. PD-168568 and tofogliflozin bind the same site but are inactive (Fig. 4B and fig. S4, C and D).

The second allosteric site is formed by the deep groove between the catalytic domains and the dimerization domain. AT7519 is the only compound in our screen that we identified bound to this site (Fig. 4C). Though it has only moderate activity, we discuss it here because this site may be a target. The chlorinated benzene ring is engaged in various van der Waals interactions to loop 107-110, Val202, and Thr292. The central pyrazole has van der Waals contacts to Ile249, Phe294 and its adjacent carbonyl group forms a hydrogen bond to the side chain of Gln110. The terminal piperidine sits on top of Asn151 and forms hydrogen bonds to the carboxylate of Asp153. This results in a displacement of loop 153-155, slightly narrowing the binding groove. The $\mathrm{C} \alpha$-atom of Tyr154 moves by $2.8 \AA$, accompanied by a conformational change of Asp153 (Fig. 4D). 
This allows hydrogen bonding to the compound and the formation of a salt-bridge to Arg298. Arg298 is crucial for dimerization (25). The mutation Arg298Ala causes a reorientation of the dimerization domain relative to catalytic domain, leading to changes in the oxyanion hole and destabilization of the $\mathrm{S} 1$ pocket by the $\mathrm{N}$ terminus. AT7519 was evaluated for treatment of human cancers (26). The potential of allosteric inhibition of $\mathrm{M}^{\text {pro }}$ through modulation of Arg298 has been independently demonstrated by mass spectrometry (27).

Our X-ray screen revealed 43 compounds binding to $\mathrm{M}^{\text {pro, }}$, with seven compounds showing antiviral activity against SARS-CoV-2. We present structural evidence for interaction of these compounds at active and allosteric sites of $\mathrm{M}^{\text {pro }}$, although we may not exclude that off-target effects played a role in the antiviral effect in cell culture, in particular for compounds with low selectivity index. Vice versa, missing antiviral activity of compounds binding clearly to $\mathrm{M}^{\text {pro }}$ in the crystal might be due to rapid metabolization in the cellular environment. Calpeptin and pelitinib showed strong antiviral activity with low cytotoxicity and are suitable for preclinical evaluation. In any case all hit compounds are valuable lead structures with potential for further drug development, especially since drug-repurposing libraries offer the advantage of proven bio-activity and cell-permeability (28).

The most active compound, calpeptin binds in the active site similar to other members of the large class of peptidebased inhibitors that bind as thiohemi-acetals or -ketals to $\mathrm{M}^{\text {pro }}$ (29). In addition to this peptidomimetic inhibitor, we discovered several non-peptidic inhibitors. Those compounds binding to the active site of $\mathrm{M}^{\text {pro }}$ contained new Michael acceptors based on $\beta$-aminoketones (tolperisone and HEAT). These lead to the formation of thioethers and have not been described as prodrugs for viral proteases. We also identified a non-covalent binder, MUT056399, blocking the active site. Besides this common active-site inhibition, we discovered compounds that inhibit the enzyme through binding at two allosteric sites of $\mathrm{M}^{\text {pro }}$.

The first allosteric site (dimerization domain) is in direct vicinity of the S1 pocket of the adjacent monomer within the native dimer. The potential for antiviral inhibition through this site is demonstrated by pelitinib. The hydrophobic nature of the residues forming the main pocket is conserved in all human coronavirus $\mathrm{M}^{\text {pro }}$ (fig. S8). Consequently, potential drugs targeting this binding site may be effective against other coronaviruses. The potential of the second allosteric site as a druggable target is demonstrated by the observed moderate antiviral activity of AT7519.

\section{REFERENCES AND NOTES}

1. L. Zhang, D. Lin, X. Sun, U. Curth, C. Drosten, L. Sauerhering, S. Becker, K. Rox, R. Hilgenfeld, Crystal structure of SARS-CoV-2 main protease provides a basis for design of improved $\alpha$-ketoamide inhibitors. Science 368, 409-412 (2020). do: $10.1126 /$ science. abb3405 Medline

2. Z. Jin, X. Du, Y. Xu, Y. Deng, M. Liu, Y. Zhao, B. Zhang, X. Li, L. Zhang, C. Peng, Y. Duan, J. Yu, L. Wang, K. Yang, F. Liu, R. Jiang, X. Yang, T. You, X. Liu, X. Yang, F. Bai, H. Liu, X. Liu, L. W. Guddat, W. Xu, G. Xiao, C. Qin, Z. Shi, H. Jiang, Z. Rao, H. Yang, Structure of Mro from SARS-CoV-2 and discovery of its inhibitors. Nature 582, 289-293 (2020). doi:10.1038/s41586-020-2223-y Medline

3. R. Hilgenfeld, From SARS to MERS: Crystallographic studies on coronaviral proteases enable antiviral drug design. FEBS J. 281, 4085-4096 (2014). doi:10.1111/febs.12936 Medline

4. J. Qiao, Y.-S. Li, R. Zeng, F.-L. Liu, R.-H. Luo, C. Huang, Y.-F. Wang, J. Zhang, B. Quan, C. Shen, X. Mao, X. Liu, W. Sun, W. Yang, X. Ni, K. Wang, L. Xu, Z.-L. Duan, Q.-C. Zou, H.-L. Zhang, W. Qu, Y.-H.-P. Long, M.-H. Li, R.-C. Yang, X. Liu, J. You, Y. Zhou, R. Yao, W.-P. Li, J.-M. Liu, P. Chen, Y. Liu, G.-F. Lin, X. Yang, J. Zou, L. Li, Y. Hu, G.W. Lu, W.-M. Li, Y.-Q. Wei, Y.-T. Zheng, J. Lei, S. Yang, SARS-CoV-2 Mpro inhibitors with antiviral activity in a transgenic mouse model. Science 371, 1374-1378 (2021). doi:10.1126/science.abf1611 Medline

5. M. Kuzikov, E. Costanzi, J. Reinshagen, F. Esposito, L. Vangeel, M. Wolf, B. Ellinger, C. Claussen, G. Geisslinger, A. Corona, D. laconis, C. Talarico, C. Manelfi, R. Cannalire, G. Rossetti, J. Gossen, S. Albani, F. Musiani, K. Herzog, Y. Ye, B. Giabbai, N. Demitri, D. Jochmans, S. D. Jonghe, J. Rymenants, V. Summa, E. Tramontano, A. R. Beccari, P. Leyssen, P. Storici, J. Neyts, P. Gribbon, A. Zaliani, Identification of Inhibitors of SARS-CoV-2 3CL-Pro Enzymatic Activity Using a Small Molecule in Vitro Repurposing Screen. ACS Pharmacol. Transl. Sci. (2021), doi:10.1021/acsptsci.0c00216

6. J. Wollenhaupt, A. Metz, T. Barthel, G. M. A. Lima, A. Heine, U. Mueller, G. Klebe, M. S. Weiss, F2X-Universal and F2X-Entry: Structurally Diverse Compound Libraries for Crystallographic Fragment Screening. Structure 28, 694-706.e5 (2020). doi:10.1016/istr.2020.04.019 Medline

7. O. B. Cox, T. Krojer, P. Collins, O. Monteiro, R. Talon, A. Bradley, O. Fedorov, J. Amin, B. D. Marsden, J. Spencer, F. von Delft, P. E. Brennan, A poised fragment library enables rapid synthetic expansion yielding the first reported inhibitors of $\mathrm{PHIP}(2)$, an atypical bromodomain. Chem. Sci. 7, 2322-2330 (2016). do:i:10.1039/C5SC03115」 Medline

8. M. M. Hann, A. R. Leach, G. Harper, Molecular complexity and its impact on the probability of finding leads for drug discovery. J. Chem. Inf. Comput. Sci. 41, 856864 (2001). doi:10.1021/ci000403i Medline

9. F. R. Ehrmann, J. Stojko, A. Metz, F. Debaene, L. J. Barandun, A. Heine, F. Diederich, S. Cianférani, K. Reuter, G. Klebe, Soaking suggests "alternative facts": Only cocrystallization discloses major ligand-induced interface rearrangements of a homodimeric tRNA-binding protein indicating a novel mode-of-inhibition. PLOS ONE 12, e0175723 (2017). doi:10.1371/journal.pone.0175723 Medline

10. H. M. Ginn, Pre-clustering data sets using cluster4x improves the signal-to-noise ratio of high-throughput crystallography drug-screening analysis. Acta

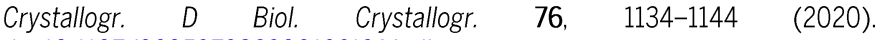
doi:10.1107/S2059798320012619 Medline

11. N. M. Pearce, T. Krojer, A. R. Bradley, P. Collins, R. P. Nowak, R. Talon, B. D. Marsden, S. Kelm, J. Shi, C. M. Deane, F. von Delft, A multi-crystal method for extracting obscured crystallographic states from conventionally uninterpretable electron density. Nat. Commun. 8, 15123 (2017). doi:10.1038/ncomms15123 Medline

12. A. L. Simplício, J. M. Clancy, J. F. Gilmer, $\beta$-aminoketones as prodrugs with $\mathrm{pH}$ controlled activation. Int. J. Pharm. 336, 208-214 (2007). do:i:10.1016/ji.jpharm.2006.11.055 Medline

13. J. Rautio, N. A. Meanwell, L. Di, M. J. Hageman, The expanding role of prodrugs in contemporary drug design and development. Nat. Rev. Drug Discov. 17, 559-587 (2018). doi:10.1038/nrd.2018.46 Medline

14. M. Altmeyer, E. Amtmann, C. Heyl, A. Marschner, A. J. Scheidig, C. D. Klein, Betaaminoketones as prodrugs for selective irreversible inhibitors of type-1 methionine aminopeptidases. Bioorg. Med. Chem. Lett. 24, 5310-5314 (2014). doi:10.1016/i.bmcl.2014.09.047 Medline 
15. S. Quasthoff, C. Möckel, W. Zieglgänsberger, W. Schreibmayer, Tolperisone: A typical representative of a class of centrally acting muscle relaxants with less sedative side effects. CNS Neurosci. Ther. 14, 107-119 (2008). doi:10.1111/j.15273458.2008.00044.x Medline

16. M. Piccart, M. Rozencweig, P. Dodion, E. Cumps, N. Crespeigne, O. Makaroff, G. Atassi, D. Kisner, Y. Kenis, Phase I clinical trial with alpha 1,3,5- triglycidyl-striazinetrione (NSC-296934). Eur. J. Cancer Clin. Oncol. 17, 1263-1266 (1981). doi:10.1016/0014-2964(81)90006-2 Medline

17. C. Ma, M. D. Sacco, B. Hurst, J. A. Townsend, Y. Hu, T. Szeto, X. Zhang, B. Tarbet, M. T. Marty, Y. Chen, J. Wang, Boceprevir, GC-376, and calpain inhibitors II, XII inhibit SARS-CoV-2 viral replication by targeting the viral main protease. Cell Res. 30, 678-692 (2020). doi:10.1038/s41422-020-0356-z Medline

18. W. Vuong, M. B. Khan, C. Fischer, E. Arutyunova, T. Lamer, J. Shields, H. A. Saffran, R. T. McKay, M. J. van Belkum, M. A. Joyce, H. S. Young, D. L. Tyrrell, J. C. Vederas, M. J. Lemieux, Feline coronavirus drug inhibits the main protease of SARS-CoV-2 and blocks virus replication. Nat. Commun. 11, 4282 (2020). doi:10.1038/s41467020-18096-2 Medline

19. M. D. Sacco, C. Ma, P. Lagarias, A. Gao, J. A. Townsend, X. Meng, P. Dube, X. Zhang, Y. Hu, N. Kitamura, B. Hurst, B. Tarbet, M. T. Marty, A. Kolocouris, Y. Xiang, Y. Chen, J. Wang, Structure and inhibition of the SARS-CoV-2 main protease reveal strategy for developing dual inhibitors against Mpro and cathepsin L. Sci. Adv. 6 , eabe0751 (2020). doi:10.1126/sciadv abe0751 Medline

20. T. Sasaki, M. Kishi, M. Saito, T. Tanaka, N. Higuchi, E. Kominami, N. Katunuma, T. Murachi, Inhibitory effect of di- and tripeptidyl aldehydes on calpains and cathepsins. J. Enzyme Inhib. 3, 195-201 (1990). doi:10.3109/14756369009035837 Medline

21. S. Escaich, L. Prouvensier, M. Saccomani, L. Durant, M. Oxoby, V. Gerusz, F. Moreau, V. Vongsouthi, K. Maher, I. Morrissey, C. Soulama-Mouze, The MUT056399 inhibitor of Fabl is a new antistaphylococcal compound. Antimicrob. Agents Chemother. 55, 4692-4697 (2011). doi:10.1128/AAC.01248-10 Medline

22. J. Tan, K. H. G. Verschueren, K. Anand, J. Shen, M. Yang, Y. Xu, Z. Rao, J. Bigalke, B. Heisen, J. R. Mesters, K. Chen, X. Shen, H. Jiang, R. Hilgenfeld, pH-dependent conformational flexibility of the SARS-CoV main proteinase (M(pro)) dimer: Molecular dynamics simulations and multiple X-ray structure analyses. J. Mol. Biol. 354, 25-40 (2005). doi:10.1016/i.jmb.2005.09.012 Medline

23. A. Wissner, E. Overbeek, M. F. Reich, M. B. Floyd, B. D. Johnson, N. Mamuya, E. C Rosfjord, C. Discafani, R. Davis, X. Shi, S. K. Rabindran, B. C. Gruber, F. Ye, W. A. Hallett, R. Nilakantan, R. Shen, Y.-F. Wang, L. M. Greenberger, H.-R. Tsou, Synthesis and structure-activity relationships of 6,7-disubstituted 4anilinoquinoline-3-carbonitriles. The design of an orally active, irreversible inhibitor of the tyrosine kinase activity of the epidermal growth factor receptor (EGFR) and the human epidermal growth factor receptor-2 (HER-2). J. Med. Chem. 46, 49-63 (2003). doi:10.1021/im020241c Medline

24. C. Erlichman, M. Hidalgo, J. P. Boni, P. Martins, S. E. Quinn, C. Zacharchuk, P. Amorusi, A. A. Adjei, E. K. Rowinsky, Phase I study of EKB-569, an irreversible inhibitor of the epidermal growth factor receptor, in patients with advanced solid tumors. J. Clin. Oncol. 24, 2252-2260 (2006). doi:10.1200/JC0.2005.01.8960 Medline

25. J. Shi, J. Sivaraman, J. Song, Mechanism for controlling the dimer-monomer switch and coupling dimerization to catalysis of the severe acute respiratory syndrome coronavirus 3C-like protease. J. Virol. 82, 4620-4629 (2008). doi:10.1128/JVI.02680-07 Medline

26. P. G. Wyatt, A. J. Woodhead, V. Berdini, J. A. Boulstridge, M. G. Carr, D. M. Cross, D. J. Davis, L. A. Devine, T. R. Early, R. E. Feltell, E. J. Lewis, R. L. McMenamin, E. F. Navarro, M. A. O'Brien, M. O'Reilly, M. Reule, G. Saxty, L. C. A. Seavers, D.-M. Smith, M. S. Squires, G. Trewartha, M. T. Walker, A. J.-A. Woolford, Identification of $\mathrm{N}$-(4-piperidinyl)-4-(2,6-dichlorobenzoylamino)-1H-pyrazole-3-carboxamide (AT7519), a novel cyclin dependent kinase inhibitor using fragment-based X-ray crystallography and structure based drug design. J. Med. Chem. 51, 4986-4999 (2008). doi:10.1021/im800382h Medline

27. T. J. El-Baba, C. A. Lutomski, A. L. Kantsadi, T. R. Malla, T. John, V. Mikhailov, J. R. Bolla, C. J. Schofield, N. Zitzmann, I. Vakonakis, C. V. Robinson, Allosteric Inhibition of the SARS-CoV-2 Main Protease: Insights from Mass Spectrometry Based Assays*. Angew. Chem. Int. Ed. 59, 23544-23548 (2020). doi:10.1002/anie.202010316 Medline
28. S. Pushpakom, F. lorio, P. A. Eyers, K. J. Escott, S. Hopper, A. Wells, A. Doig, T. Guilliams, J. Latimer, C. McNamee, A. Norris, P. Sanseau, D. Cavalla, M. Pirmohamed, Drug repurposing: Progress, challenges and recommendations. Nat. Rev. Drug Discov. 18, 41-58 (2019). doi:10.1038/nrd.2018.168 Medline

29. Y. Liu, C. Liang, L. Xin, X. Ren, L. Tian, X. Ju, H. Li, Y. Wang, Q. Zhao, H. Liu, W. Cao, $X$. Xie, D. Zhang, Y. Wang, Y. Jian, The development of Coronavirus 3C-Like protease $\left(3 \mathrm{CL}^{\text {pro }}\right.$ ) inhibitors from 2010 to 2020. Eur. J. Med. Chem. 206, 112711 (2020). doi:10.1016/j.ejmech.2020.112711 Medline

\section{ACKNOWLEDGMENTS}

We acknowledge Deutsches Elektronen-Synchrotron (DESY, Hamburg, Germany), a member of the Helmholtz Association HGF, for the provision of experimental facilities. Parts of this research were carried out at PETRA III at beamline P11. Further MX data were collected at beamline P13 and P14 operated by EMBL. We thank the DESY machine group, in particular Mario Wunderlich, Kim Heuck, Arne Brinkmann, Olaf Goldbeck, Jürgen Haar, Torsten Schulz, Gunnar Priebe, Maximilian Holz, Björn Lemcke, Klaus Knaack, Oliver Seebauer, Philipp Willanzheimer, Rolf Jonas, and Nicole Engling. We thank Thomas Dietrich, Simon Geile, Filip Guicking, Heshmat Noei, and Tim Pakendorf from DESY, and Bianca Di Fabrizio and Sebastian Kühn from BNITM for assistance. This research was supported in part through the Maxwell computational resources operated at DESY. We acknowledge the use of the XBI biological sample preparation laboratory at European XFEL, enabled by the XBI User Consortium. Funding: We acknowledge financial support from the EXSCALATE4COV EU-H2O2O Emergency Project (101003551), the Cluster of Excellence "Advanced Imaging of Matter" of the Deutsche Forschungsgemeinschaft (DFG) - EXC 2056 - project ID 390715994, the Helmholtz Association Impulse and Networking funds (projects ExNet-0002 and InternLabs-0011 "HIR3X"), the Federal Ministry of Education and Research (BMBF) via projects 05K16GUA, 05K19GU4, 05K20BI1, 05K20FL1, 16GW0277 and 031B0405D), and the Joachim-Herz-Stiftung Hamburg (project Infecto-Physics). CE and MR acknowledge financial support from grant-No. HIDSS-0002 DASHH (Data Science in Hamburg - HELMHOLTZ Graduate School for the Structure of Matter). RC is supported by DFG grants INST 187/621-1 and INST 187/686-1. DT is supported by the Slovenian Research Agency (ARRS; research program P1-0048, Infrastructural program 10-0048). BS was supported by an Exploration Grant from the Boehringer Ingelheim Foundation. The Heinrich Pette Institute, Leibniz Institute for Experimental Virology was supported by the Free and Hanseatic City of Hamburg and the Federal Ministry of Health. CU and BK were supported by EU Horizon 2020 ERC StG-2017 759661, BMBF RTK Struktur 01KI20391, BMBF Visavix 05K16BH1 and the Leibniz Association SAW-2014-HPI-4 grant. Author contributions: SeG, PR, YFG, WB, PG, ARB, RC, DT, AZ, HNC, ARP, CB, AM designed research. SeG, PR, TJL, WH, HNC, ARP, CB, AM wrote manuscript. SeG, PR, JL, FHMK, SM, WB, ID, BS, HGie, BNB, MB, PLX, NW, HA, NU, SF, BAF, MS, HB, JK, GEPM, ARM, FG, VH, PF, MW, ECS, PM, HT, TB participated in sample preparation. PR performed crystallization experiments. SeG, PR, JL, TJL, OY, SS, AT, MGr, HF, FT, MGa, YG, CFL, SA, AP, GB, DVS, GP, TRS, IB, SP performed X-ray data collection. TJL, HGin, DO, OY, LG, MD, TAW, FS, CR, DM, JZD, IK, CS, RS, HUH, DCFM contributed to X-ray data management. SeG, PR, JL, TJL, HGin, FHMK, WE, DO, AH, VS, JH, JM, JB, JW, CF, MSW, AC, DT, WH, AM performed X-ray data analysis. KL, BK, CU, RC performed and analyzed MS experiments. YFG, BEP, StG performed and analyzed antiviral activity assays. PG, BE, MK, MGA, SN, CG, LZ, XS, KK, AU, JL, RH performed and analyzed ligand binding studies and protein activity assays. CE, JPZ, MR performed computational binding studies. Competing interests: MR is stakeholder of BioSolvelT GmbH, licensor of the software HYDE. Data and materials availability: The coordinates and structure factors for all described crystal structures of SARS-CoV-2 Mpro in complex with compounds are deposited in the PDB with accession codes 6YNQ, 6YVF, 7A1U, 7ABU, 7ADW, 7AF0, 7AGA, 7AHA, 7AK4, 7AKU, 7AMJ, 7ANS, 7AOL, 7AP6, 7APH, 7AQE, 7AQI, 7AQJ, 7AR5, 7AR6, 7ARF, 7AVD, 7AWR, 7AWS, 7AWU, 7AWW, 7AX6, 7AXM, 7AX0, 7AY7, 7B83 and 7NEV. Code used in this analysis has been previously published (10). The code for forcing adherence to the Wilson distribution is included in the Vagabond refinement package (https://vagabond.hginn.co.uk/) under a GPLv3 license. Compounds from the Fraunhofer IME Repurposing collection were obtained from the Fraunhofer 
Institute for Molecular Biology and Applied Ecology under a Material Transfer Agreement. Compounds from the Safe-in-man Library were kindly provided by Dompé Farmaceutici S.p.A. Other materials are available from SeG or AM upon request. This work is licensed under a Creative Commons Attribution 4.0

International (CC BY 4.0) license, which permits unrestricted use, distribution, and reproduction in any medium, provided the original work is properly cited. To view a copy of this license, visit

https://creativecommons.org/licenses/by/4.0/. This license does not apply to figures/photos/artwork or other content included in the article that is credited to a third party; obtain authorization from the rights holder before using such material.

\section{SUPPLEMENTARY MATERIALS}

science.sciencemag.org/cgi/content/full/science.abf7945/DC1

Materials and Methods

Supplementary Text

Figs. S1 to S9

Tables S1 to S7

References (30-54)

MDAR Reproducibility Checklist

20 November 2020; accepted 29 March 2021

Published online 2 April 2021

$10.1126 /$ science.abf7945 

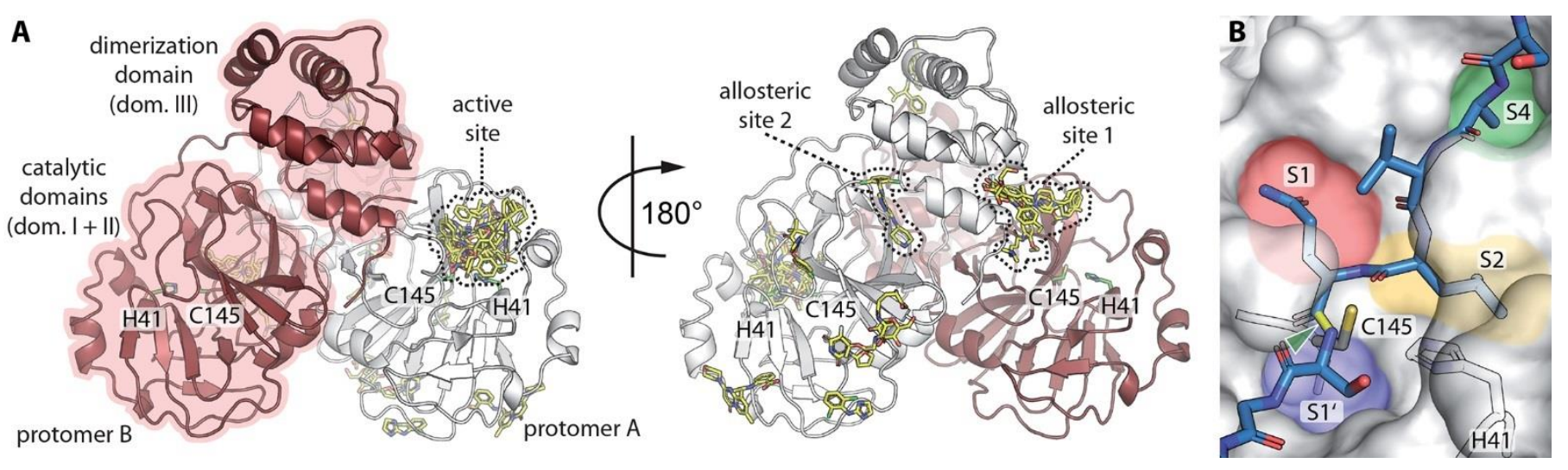

Fig. 1. X-ray screening of drug-repurposing libraries reveals compound binding sites distributed across the complete $\mathrm{M}^{\text {pro }}$ surface. (A) Schematic drawing of $\mathrm{M}^{\text {pro }}$ dimer structure. Protomer $\mathrm{A}$ in white, protomer $\mathrm{B}$ in red. For clarity, the 29 binding compounds (yellow sticks) are only depicted on one of the two protomers. Catalytic residues $\mathrm{H} 41$ and Cys145, active site and two allosteric drug binding sites are highlighted. (B) Close-up view of active site with peptide substrate bound (blue sticks), modeled after SARS-CoV Mpro (PDB 2Q6G). Scissile bond is indicated in yellow and with green arrow. Substrate binding pockets S1', S1, S2 and S4 are indicated by colors. 

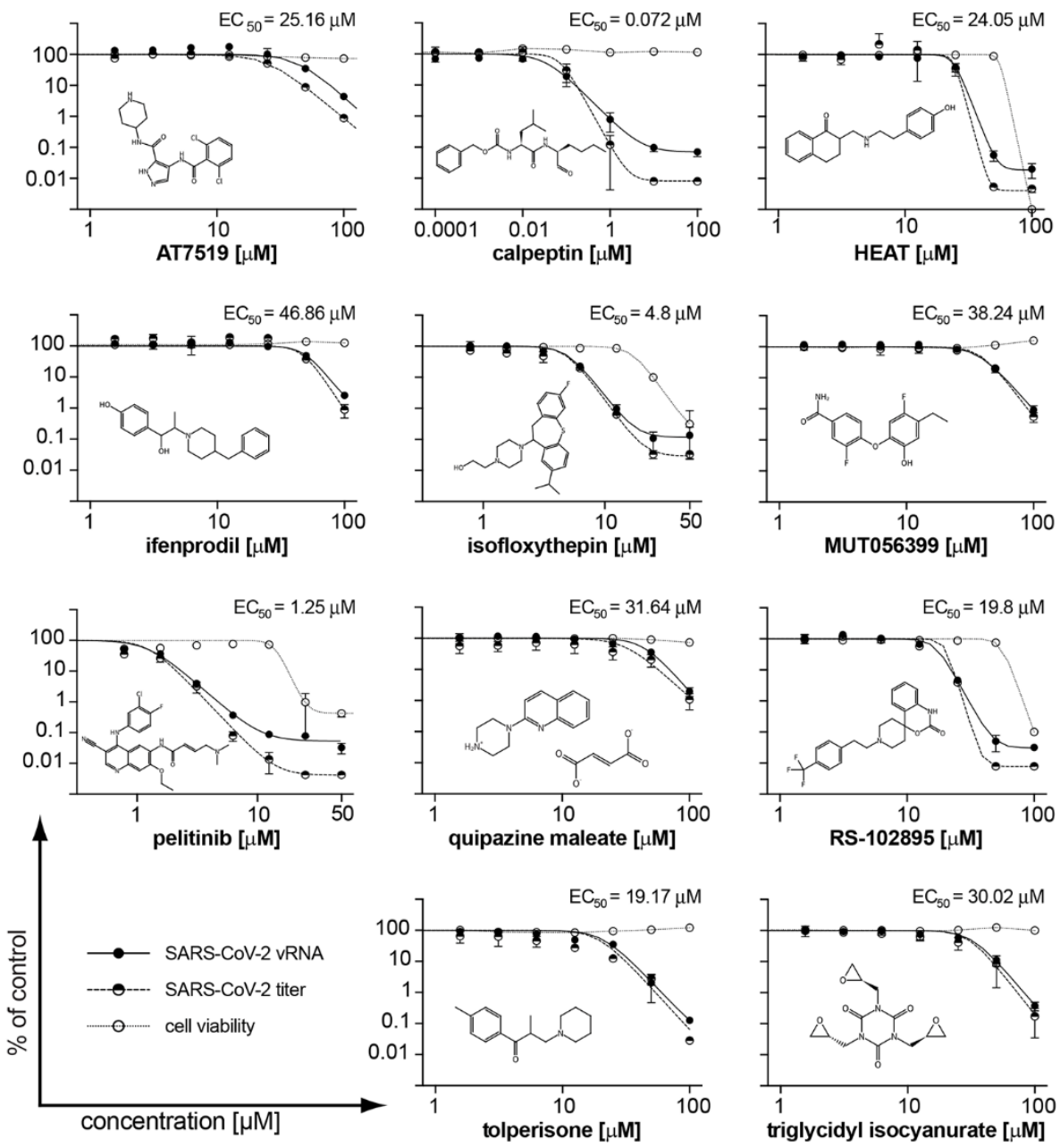

Fig. 2. Effect of selected compounds on SARS-CoV-2 replication in Vero E6 cells. The vRNA yield (solid circles), viral titers (half-solid circles), and cell viability (empty circles) were determined by RT-qPCR, immunofocus assays, and the CCK-8 method, respectively. $\mathrm{EC}_{50}$ for the viral titer reduction is shown. Individual data points represent mean \pm SD from three independent replicates in one experiment. 


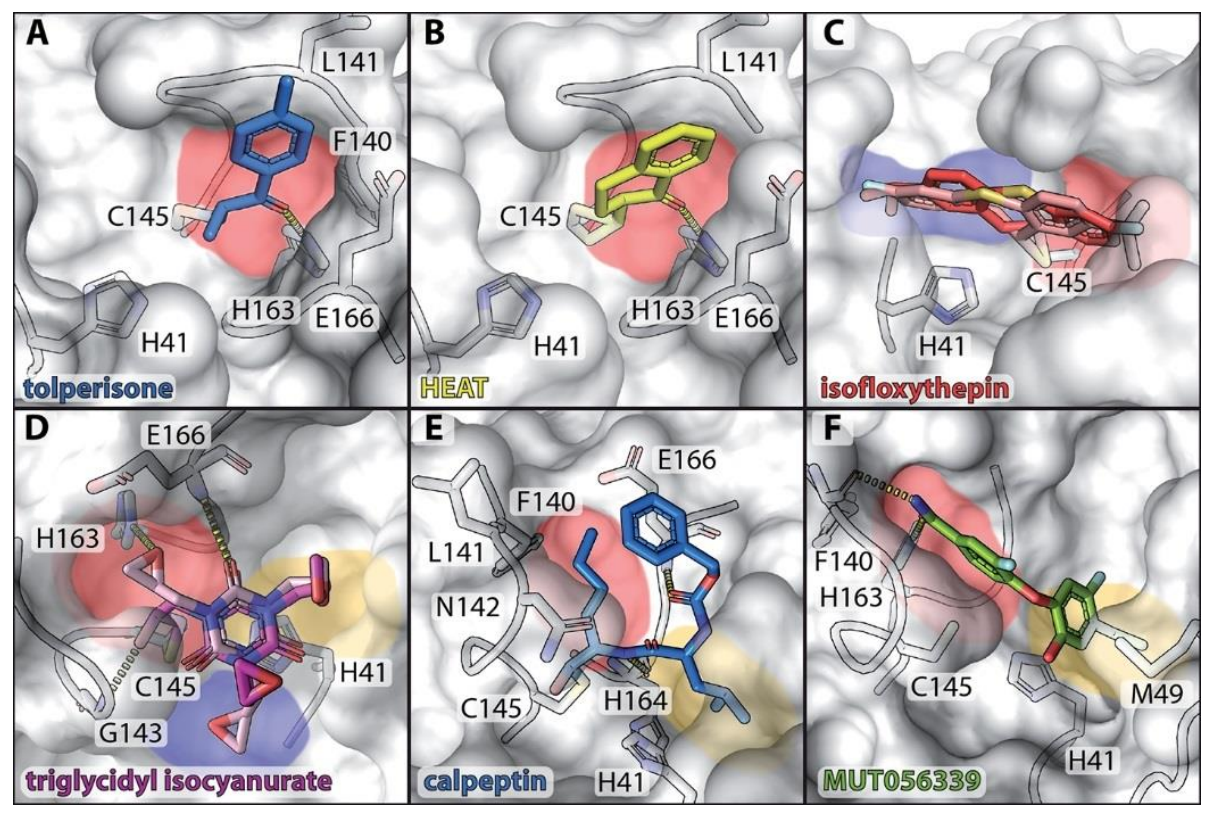

Fig. 3. Covalent and non-covalent binders in the active site of $\mathrm{M}^{\text {pro }}$. Bound compounds are depicted as colored sticks while the surface of $\mathrm{M}^{\text {pro }}$ is shown in grey with selected interacting residues as sticks. Substrate binding pockets are colored as in Fig. 1. Hydrogen bonds are depicted by dashed lines. (A) tolperisone. (B) HEAT, (C) isofloxythepin, (D) triglycidyl isocyanurate, (E) calpeptin, (F) MUT056399. 


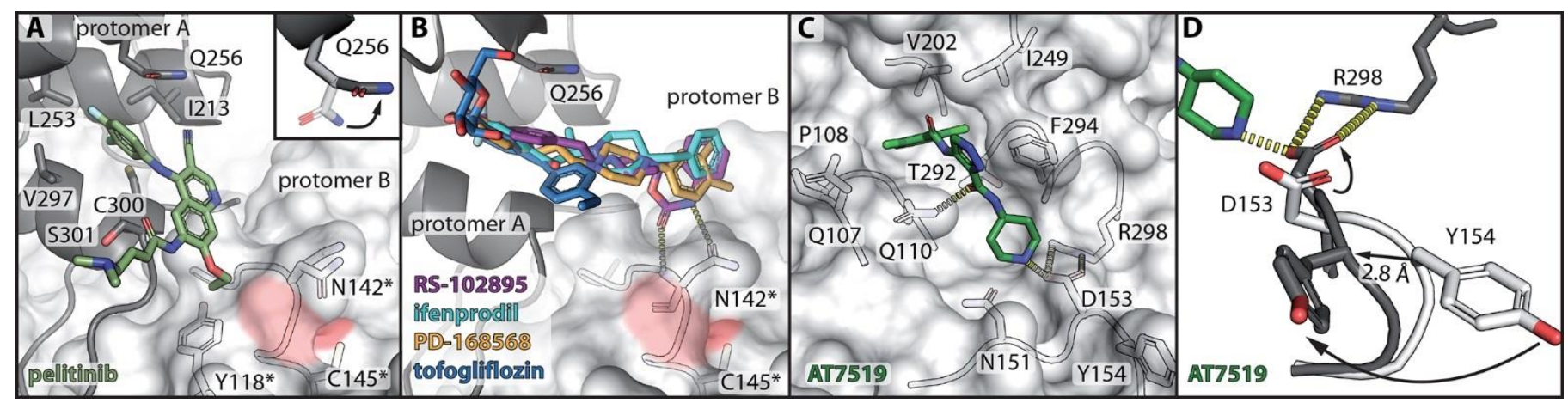

Fig. 4. Screening hits at allosteric sites of $M^{\text {pro }}$. (A) Close up view of the binding site in the dimerization domain (protomer A, grey cartoon representation), close to the active site of the second protomer (protomer B, surface representation) in the native dimer. Residues forming the hydrophobic pocket are indicated. Pelitinib (dark green) binds to the C-terminal $\alpha$-helix at Ser301 and pushes against Asn142 and the $\beta$-turn of the pocket S1 of protomer B (residues marked with an asterisk). The inset shows conformational change of Gln256 (grey sticks) compared to $\mathrm{M}^{\text {pro }}$ apo structure (white sticks). (B) RS-102895 (purple), ifenprodil (cyan), PD-168568 (orange) and tofogliflozin (blue) occupy the same binding pocket as pelitinib. (C) AT7519 occupies a deep cleft between the catalytic and dimerization domain of $\mathrm{M}^{\text {pro }}$. (D) Conformational changes in the AT7519 bound $\mathrm{M}^{\text {pro }}$ structure (grey) compared to the apo structure (white). 